\title{
Rhetorische Kompetenz bei polnischen Studierenden in der Fremdsprache Deutsch im universitären Kontext
}

\section{Zum Stellenwert der Ausbildung der rhetorischen Kompetenz. Einleitende Bemerkungen}

Vortragen (Präsentieren/Referieren) sachbezogener Inhalte und Diskutieren, Debattieren sowie Verhandeln stellen grundlegende kommunikative makrostrukturelle Muster dar, die der institutionellen Kommunikation in verschiedenen Kontexten zuzurechnen sind. Diese rhetorischen Fähigkeiten und Fertigkeiten erleichtern spätestens auf der Universitätsebene einen effektiven Wissenserwerb und verbessern zugleich allgemeine Studierfähigkeiten. Darüber hinaus fördern sie die Optimierung der Kommunikationsprozesse auf der weit gefassten Berufsebene. Der Erwerb rhetorischen Wissens und Könnens erhöht somit persönliche Erfolgschancen bei der Jobsuche bzw. einem Stellenwechsel, sodass „Investitionen in diesen Bereich [...] als Investitionen in die berufliche Karriere gesehen" (Jakobs 2009:2384) werden (vgl. auch Forster 1995:16). Aus diesem Grund machen Rhetorikseminare schon seit vielen Jahren einen festen Bestandteil der beruflichen Fort- und Weiterbildung in Deutschland aus. Das Themenspektrum reicht von eher allgemeinen Kommunikationstrainings, die hauptsächlich auf Vortragssituationen angelegt sind, bis hin zu den speziellen, an beruflich relevanten Aufgaben- und Situationstypen (Interaktion, Kooperation, Konflikt) orientierten Angeboten. Im Allgemeinen sollen sie den Teilnehmern zu wirkungsvollem Sprechen/Reden verhelfen. Derartige Kommunikationstrainings werden in den letzten zehn Jahren zunehmend auch in Polen implementiert, wobei jedoch, wenn es sich um Trainings im Bereich Deutsch als Fremdsprache handelt, Aspekte der interkulturellen Kommunikation, wie etwa erwartungswidrige Wirkung der Sprecherper- 
son in der Fremdsprache bzw. des fremden Akzents, gar nicht mit berücksichtigt werden.

Angesichts einer hohen Nachfrage nach professioneller Rhetorik gehört die Übermittlung der rhetorischen (Schlüssel-)Kompetenz, zumindest an deutschen Universitäten, zum breiten Angebot an zahlreichen Lehrveranstaltungen im Rahmen der überfachlichen Allgemeinen Schlüsselqualifikationen (ASQ), wie das z.B. an der Martin-Luther-Universität in Halle-Wittenberg (http://www. asq.uni-halle.de/), an der Justus-Liebig-Universität in Giessen (https://www. uni-giessen.de/cms/fbz/zentren/zfbk/afk/kursangebot/zfbk), an der Ernst-MoritzArndt-Universität in Greifswald (http://www.rsf.uni-greifswald.de/studium/propae-deutik.html) oder an der Friedrich-Schiller-Universität in Jena (https://www. uni-jena.de/Schluesselqualifikationen_p_151795.html) der Fall ist. Da aber die Rhetorikkurse nur fakultativ gewählt werden, ist auf diesem Gebiet nicht selten eine Ausbildungslücke zu verzeichnen. Auch in der Muttersprache verfügen die potenziellen Kommunikationsinteraktanten nicht immer über ausreichendes Wissen um Wirkungsphänomene und Wirkungskriterien. Ein besonders großer Nachholbedarf in diesem Bereich ist auch in den germanistischen Studiengängen in Polen anzumelden, wo angewandte Rhetorik laut eigener Recherche tatsächlich nur ausnahmsweise als Lehrfach angeboten wird, wie z.B. an der Universität Łódź. Ebenso in Ausnahmefällen wird sie zum integralen Bestandteil der fachbzw. sprachbezogenen Ausbildung. Diese Tatsache mag der Bildungstradition in Bezug auf die Entwicklung der rhetorischen Kompetenz in der Muttersprache geschuldet sein. Die Vermittlung rhetorischer Fähigkeiten und Fertigkeiten wird als Bildungsziel beispielsweise im ministerialen Curriculum für den muttersprachlichen Polnischunterricht eher unvollständig beschrieben und somit in der Praxis inkonsequent wie auch uneinheitlich verfolgt (ausführlicher dazu vgl. Grzeszczakowska-Pawlikowska 2011:221-224). Nichtsdestotrotz wird die Fähigkeit zur rhetorischen Kommunikation in der Fremdsprache Deutsch im universitären Unterrichtsalltag des Öfteren schlechthin vorausgesetzt. Ausgehend von der Annahme eines möglichen Einflusses der jeweiligen Bildungstradition auf die Textproduktion in der Zielsprache (vgl. Eßer 1997:82, Clyne 1993:10) ist jedoch eine derartige Erwartungshaltung nicht begründet. Wenn also polnische Studenten beispielsweise mit der Aufgabe konfrontiert werden, vor ihrer Seminargruppe ein Referat in der Fremdsprache Deutsch zu halten, ohne dass sie darauf fundiert vorbereitet werden, stoßen sie auf eine Reihe rhetorischer Schwierigkeiten. Diese manifestieren sich in einer äußerst großen Kompetenzlücke, die erst mit entsprechenden Redetrainings systematisch zu füllen wäre. Hinzu kommen, so die eigene Lehrerfahrung, weitere Probleme, wie etwa das jeweilige Sprachniveau der Studierenden, deren unzureichender Umgang mit Sprechangst, persönliche Motivationen etc., die bei der Entwicklung der rhetorischen Kompetenz als Schlüsselfertigkeit in der Fremdsprache nicht ohne Beachtung bleiben sollten. In der universitären Lehr-Lern-Praxis sind all diese Aspekte zielgerichtet zu behandeln, 
da sie das rhetorische Können der nicht-muttersprachlichen Sprecher des Deutschen unter Umständen potenziell weitgehend abschwächen. Welche Faktoren es konkret sind, soll im weiteren Teil des vorliegenden Beitrags anhand empirischer Daten ausführlicher dargestellt werden. Zunächst sollen einige für diesen Kontext relevante Begriffe näher erläutert werden.

\section{Kompetenzorientierung in der akademischen Bildung. Der Kompetenzbegriff}

Ein zentrales Ziel der Bologna-Reform (1999) war (und ist nach wie vor) die Sicherung der Beschäftigungsfähigkeit europäischer AkademikerInnen als Fähigkeit einer Person „auf der Grundlage ihrer fachlichen und überfachlichen Handlungskompetenzen ihre Arbeitskraft auf einem sich [...] veränderten Markt anbieten zu können und damit Zugang zum Erwerbsleben zu erhalten bzw. sich darin erfolgreich bewegen zu können“ (Greinert 2008:10). Die Forderung nach Beschäftigungsfähigkeit geht dabei mit einer allgemeinen Schwerpunktverschiebung im Bildungsbereich einher - vom traditionellen Berufskonzept mit Erwerb einer fachspezifischen Befähigung zu einem umfassenden employability-Konstrukt, in dem der Akzent vor allem auf ,die dauerhafte Bereitschaft zur Anpassung des individuell Vorhandenen an das arbeitsmarktseitig Geforderte" (Kraus 2007:5) gelegt wird. Eine naheliegende Konsequenz dieser Forderung ist die Erweiterung der Bildungsziele zwecks weitgehender Kompetenzorientierung in Studium und Lehre. Dementsprechend sollen Studierende nicht nur zum wissenschaftlichen Denken befähigt werden, das im Umgang mit dem vermittelten Wissen (Reflektieren, Überprüfen, Hinterfragen etc.) Ausdruck findet. Zugleich sollte auch eine systematische Vorbereitung der angehenden AkademikerInnen auf ein breites berufliches Tätigkeitsfeld erfolgen. Dabei ist allerdings nicht mehr so sehr ein konkreter, spezifischer (akademischer) Beruf, sondern eine weit gefasste Beruflichkeit anzustreben (vgl. Schaper 2012:8-9). Mit anderen Worten: In bildungspolitischer Hinsicht kommt es nicht vorrangig auf die Stoff- bzw. Wissens-Orientierung, sondern auf die Befähigung zu komplexen Handlungen an, indem fachübergreifende, beruflich verwertbare Kompetenzen, begleitet durch die Entwicklung individueller Erwerbsorientierung, vermittelt werden (sollen). Die Aneignung bzw. die Vermittlung wissenschaftlich-fachbezogener Inhalte sollte also kein übergeordnetes und nicht das einzige Ziel des akademischen Unterrichts im europäischen Hochschulraum der Gegenwart darstellen. Vielmehr handelt es sich beim Konzept der employability insgesamt um ein Zusammenspiel von Fähigkeiten zur berufsfeldübergreifenden, situationsbezogenen und innovativen Lösung beruflicher Aufgaben und Probleme sowie die Fähigkeit zum selbstorganisierten lebenslangen Lernen in konkreten beruflichen Situationen (vgl. Schwar- 
ze 2011:56). Damit kann unmittelbar an den Begriff der Kompetenzen angeknüpft werden, die im vorliegenden Aufsatz in Anlehnung an Weinert aufgefasst werden als ,die bei Individuen verfügbaren oder durch sie erlernten kognitiven Fähigkeiten und Fertigkeiten, bestimmte Probleme zu lösen, sowie die damit verbundenen motivationalen, volitionalen und sozialen Bereitschaften und Fähigkeiten, die Problemlösungen in variablen Situationen erfolgreich und verantwortungsvoll nutzen zu können“" (Weinert 2001:27-28). Die einzelnen Kompetenzen lassen sich durch beobachtbare Handlungen nachweisen, sodass diesbezüglich die Handlungskomponente von großer Relevanz ist. Kompetenzen selbst sind allerdings nicht mit beobachtbarem Handeln gleichzusetzen, sondern sie stellen „Handlungspotenziale [dar], die vor allem in variablen Situationen konstruktiv genutzt werden können“ (Schwarze 2011:59). Bei Kompetenzen kommt es also jeweils auf bestimmte Dispositionen an, Entsprechendes zu leisten, wobei diese sich erst in der Anwendung von Wissen konkretisieren. Daher spielen auch der jeweilige Kontext bzw. die Kontextgebundenheit eine bedeutende Rolle. Demnach besteht die Entwicklung der jeweiligen Kompetenzen im Sammeln von Erfahrungen in entsprechenden Situationen bzw. Kontexten (vgl. die Übersicht bei Schaper 2012:12-23). Darüber hinaus sind Kompetenzen generativ, d.h. sie können eine unbestimmte Menge von Handlungen regelbasiert erzeugen; sie sind individuell zuzuschreiben, beinhalten ein Potenzial bzw. Repertoire von Alternativen und lassen sich normativ im Sinne einer Unterscheidung zwischen kompetenten und inkompetenten Handlungen abgrenzen (vgl. Barthel 2007:229). Als normativer Begriff beinhaltet Kompetenz ein Können im „Sinne einer ,geglückten Performanz““ (Deppermann 2009:17).

Im Allgemeinen dient das Kompetenz-Konstrukt zur Erfassung menschlicher Leistungen in den verschiedenen Lebensbereichen. Der unterschiedliche Charakter dieser Leistungen setzt ein breites Spektrum mentaler Prozesse und Kapazitäten voraus, die auch für die Persönlichkeitsentwicklung ausschlaggebend sind. Die jeweilige Kompetenz ist dabei nicht isoliert als reines Faktenwissen anzusehen, sondern als Zusammensetzung verschiedener Facetten, die sich z.T. weitgehend decken können. Dazu gehören z.B. nach Klieme et al. (2007:73) Erfahrung, Handeln, Motivation, (prozedurales) Können, Verstehen und Wissen. Diese Betrachtungsweise von Kompetenz ist ebenfalls für das Verständnis der rhetorischen Kompetenz grundlegend, auf die im darauffolgenden Kapitel ausführlicher eingegangen wird.

\section{Rhetorische Kompetenz als Schlüsselkompetenz}

Die Auffassung des Rhetorischen als intentionales, kommunikativ-dialogisches Sprech- und Hörhandeln in verschiedenen Gesprächs- und Redeformen stellt einen geeigneten Ansatzpunkt für die Auffassung der rhetorischen Kompetenz 
dar. Gemeint ist damit die umfassende kommunikative Handlungsfähigkeit zur zweckorientierten Abwicklung intendierter sozialer Interaktionen durch wirksames Miteinandersprechen (Gespräch) und Zueinanderreden (Rede) sowie zur bewussten Reflexion über die jeweiligen Kommunikationsprozesse, darunter das eigene Verhalten und Handeln. Durch die erkennbare Integration von Handlungs- und Reflexionswissen lässt sich der hier erläuterte Begriff der rhetorischen Kompetenz zugleich auch mit dem traditionellen Rhetorik-Begriff als Téchne (Kunstlehre, d.h. lehr- und lernbares System, und Kunstfertigkeit als individuelle Fähigkeit zur Anwendung dieses Systems) zusammenführen (vgl. Bose/Schwarze 2007:11). Den Kern der rhetorischen Kompetenz im aktuellen Verständnis macht allerdings nicht, wie in der Antike, die Kunst des schönen Redens aus. In den Rhetorik-Trainings der Gegenwart soll vor allem auf die Befähigung des Menschen zur aktiven eigenverantwortlichen Teilnahme an den rhetorischen Sprechsituationen fokussiert werden. Von bedeutender Relevanz ist dabei eine Reihe von Fragen, z.B. nach der Wirkung eigenen Verhaltens/Handelns, nach dessen Wahrnehmung und Interpretation durch andere, nach möglichen Reaktionen, nach Ursachen für Kommunikationsbarrieren etc. (vgl. auch Slembek 1999:88). Im Hinblick darauf erscheint rhetorische Kompetenz als wesentliche Voraussetzung für den optimalen Ablauf einer störungsfreien Kommunikation.

Bei der Vermittlung rhetorischer Kompetenz ist im Allgemeinen weit gefasste Gesprächs- und Redekompetenz im Sinne sog. intersubjektiver Komplexprozesse anzustreben, d.h. hinzuarbeiten auf die Fähigkeit zur Gesprächsführung und zum Halten von Reden (Vortragen/Präsentieren/Referieren). Zugleich kommt es auch auf eine zweckgerichtete Arbeit am Sprechdenken und Hörverstehen im Sinne sog. intrasubjektiver Elementarprozesse an, die für komplexe kommunikative Vorgänge konstitutiv sind. Vor dem Hintergrund der hier vertretenen Annahme der Multimodalität sprechsprachlicher Verständigung - deren Totalität hinsichtlich sprachlicher, physischer und physikalisch-materieller, sprich: sprechsprachlich-körperlicher, etc. Dimension - verdienen in einem Rhetorik-Training darüber hinaus auch performative Handlungsfähigkeiten zum angemessenen Sprech- und Körperausdruck entsprechende Aufmerksamkeit. Von Bedeutung ist schließlich argumentative Kompetenz, d.h. die Fähigkeit zum Einsatz argumentativer Strukturen und Strategien, die hier als Variante von Gesprächs- und Redekompetenz begriffen wird (vgl. Grundler 2011:79). Dazu gehören im Einzelnen u.a. Themenund Aufgabenbezug, Kooperativität, Partnerbezug sowie Angemessenheit, die um explizites Wissen, Ziel-Mittel-Relationen sowie um den Überzeugungs- bzw. Erfolgsbezug zu erweitern sind (vgl. Schwarze/Bose 2013:77). Dies gilt im selben Maße für Gesprächsführung ebenso wie für Vortragssituationen. Rhetorisches Sprechen bedeutet also nicht nur angemessener Einsatz grammatikalischer Strukturen, phonetische Verständlichkeit und einen Blick, der gelegentlich ins Publikum geworfen wird. Vielmehr soll in der Arbeit an der rhetorischen Kompetenz, wie gerade erläutert, auf zahlreiche wesentliche Aspekte sprechsprachlichen kom- 
munikativen Handelns Bezug genommen werden, die jedoch in der universitären Sprachpraxis, so die eigene Beobachtung, kaum Beachtung finden.

$\mathrm{Zu}$ fokussieren ist letztendlich bei der Vermittlung der rhetorischen Kompetenz auf den gegenseitigen intentionalen Austausch der Kommunikationspartner zum Zweck gemeinsamer Aufgabenbewältigung, wobei potenzielle Konflikte grundsätzlich nicht vermieden, sondern mittels argumentativer und kooperativer Verfahrensweisen ausgetragen werden sollen. In Anbetracht dessen ist die Forderung nach einer expliziten Einbeziehung rhetorischer Fähigkeiten und Fertigkeiten in das Konzept der Schlüsselkompetenzen naheliegend (vgl. auch Schwarze 2007:238, Pabst-Weinschenk 2009:7). Als Sozialkompetenz kann rhetorische Kompetenz sogar den Rang einer übergeordneten Begrifflichkeit für die weiteren sozial determinierten Kommunikations-, Kooperations- und Konfliktfähigkeiten (vgl. Knauf 2001:47) erhalten.

Im Hinblick auf die einleitend formulierte Problemstellung bezüglich potenzieller Schwierigkeiten, mit denen polnische Germanistikstudierende beim Halten von Seminarreferaten konfrontiert werden, soll nun schlussendlich auf Redekompetenz als rhetorische Teilkompetenz ausführlicher eingegangen werden. Die folgenden theoretischen Ausführungen sollen als Grundlage für die Darstellung eigener empirischer Untersuchungen sowie deren Ergebnisse dienen (s. Kap. 5). Ausgegangen wird von einer Auffassung der Rede als rhetorischer Gattung.

\section{Redekompetenz}

Im Allgemeinen gilt Rede als „Oberbegriff für alle Prozesse, in denen eine Person zu einer anderen oder mehreren anderen Personen spricht" (Gutenberg 2001a:81). Aus sprechwissenschaftlich-rhetorischer Sicht ist sie jedoch auf kommunikative Symmetrie angelegt (vgl. z.B. Pabst-Weinschenk 2004:102; auch 118). Das heißt, das Grundmuster Frage-Antwort ist nicht nur für den Ablauf von Gesprächen bestimmend. Zwecks intendierter Verständigung hat der Redner, genauso wie in einem Gespräch, die Fragen und die Position des Zuhörers zu berücksichtigen (vgl. Pabst-Weinschenk 2004:18). Den Bezug zum Gegenüber (den Hörerbezug), der sich u.a. in der Perspektivenübernahme manifestiert, erfordert dabei nicht nur die konkrete Sprechsituation (vgl. Schwarze/ Bose 2013:77). Vielmehr soll bereits in der Vorbereitungsphase einer Rede (bei der Behandlung des jeweiligen Themas) darauf gezielt werden, "die situativ und rollengemäß erfasste Hörerschaft mitarbeiten (kooperieren) [zu] lassen“" (Bartsch 1990:41). Dies wird zum Maßstab für all jene Fälle, in denen man Sprechen grundsätzlich nicht als Konkurrenzsituation auffasst, sondern „eine kooperative Einstellung vertritt“" (Pabst-Weinschenk 2009:111). In der Redepraxis kommt es also insgesamt auf hörerorientiertes Sprechen an. In dem Sinne stellt Rede ein gemeinschaftliches Kommunikationsereignis dar. Die Fähigkeit zur Übernahme der Perspektive des Anderen gilt dabei als eine der kognitiven 
Grundfähigkeiten des Menschen und zugleich als eine der fundamentalen Voraussetzungen für die Erzielung der beabsichtigten Rede-Wirkungen.

In Anbetracht dessen wird Rede im Weiteren nicht als Monolog betrachtet, obwohl in der kommunikativen Wirklichkeit in der Regel tatsächlich nur eine Person spricht, sondern als Dialog. Im Unterschied zum unmittelbar (real) Dialogischen ist jedoch mit Rede eine virtuell-dialogisch vollzogene Kommunikationsform, d.h. das latent Dialogische gemeint. In der Rede wird der Sprecherwechsel „temporär suspendiert“ (Schwarze/Bose 2013:77) und das Rederecht liegt grundsätzlich fest, die Rollenverteilung (Redner vs. Zuhörer) muss allerdings im Voraus von allen Beteiligten akzeptiert werden. Darüber hinaus ist das mit der Fragehaltung des Redners korrespondierende Feedback, bis auf gelegentliche Zwischenrufe, nonverbal (mimisch, gestisch, phonisch). Äußerungen aus der Zuhörerschaft während der Rede sind dagegen als Ausnahmen zu werten - anders als bei Gesprächen, in denen die Sprecher- und Hörerrolle durchgehend austauschbar sind und das verbale sowie nonverbale Feedback das Sprechhandeln von beiden Seiten steuern. Erst beim nichtvorhandenen Hörerbezug bzw. bei fehlender Bereitschaft, „die Rückmeldungen der Hörerschaft sprechdenkend einzuarbeiten, erstarrt die Rede [...] zum Monolog“" (Geißner 1986:125).

Im Hinblick auf die umfassende Dialogizität der zwischenmenschlichen Kommunikation ist also Rede, analog zum Gespräch, zusammenfassend aufzufassen als „virtuell-dialogische, intendierte Verständigungshandlung eines Redners für meist mehrere Hörer mit dem Ziel, etwas zur gemeinsamen Sache zu machen, um gemeinsames (mentales oder reales) Handeln zu ermöglichen“" (Meyer 2013:121). Im Hinblick darauf wird nun Redekompetenz als Fähigkeit, „, handelnd das Ziel durch die Rede [zu] erreichen“" (vgl. Barthel 2007:230) definiert. Gemeint ist damit die Befähigung eines Redners zum kommunikativen Handeln zwecks Kooperation sowie verbaler Konfliktlösung unter Einbeziehung aktueller Wissensbestände. Von Relevanz ist zudem der Wirkungseffekt, den der Redner bei der Zuhörerschaft zu erzielen beabsichtigt. Insgesamt kommt also bei Redekompetenz, um es noch einmal an dieser Stelle zu betonen, nicht auf die bloße Redekunst an, sondern auf das intendierte, handlungsauslösende, kooperative sowie das auf Verständigung zielende Reden. Somit handelt es sich nicht ausschließlich bzw. gar nicht um die Durchsetzung der eigenen Meinung und/oder um das Bewegen zu einer bestimmten Handlung, ohne dass die Lage der Hörer, deren Erwartungen sowie deren Erfahrungen bezüglich der gegebenen Sprechsituation berücksichtigt werden. Vielmehr kommt es auf eine Reihe trainierbarer Fähigkeiten an, die als potenzielle Wirkungsdeterminanten das wirksame Reden in unterschiedlichen Ausprägungen, darunter auch in der institutionellen Lehr-Lern-Kommunikation in Gestalt studentischer Seminarreferate, jeweils fördern oder abschwächen können. Die möglichen Wirkungsvariablen gehören dabei zu unterschiedlichen Bereichen, wie etwa dem des Sprechers (dessen Stimme und Sprechweise, dessen äußere Erscheinung einschließlich nonverbalen Verhaltens, dessen soziale 
Situation etc.), der Äußerung (die kommunikative Funktion der Aussage, deren Gliederung und -aufbau, Argumentation etc.) sowie dem Bereich der Rezipienten (deren Aufmerksamkeitsverlauf und Behaltensleistung, allgemeine Prädispositionen etc.) (vgl. Stock 1991:19-20). Die einzelnen Wirkungsvariablen werden jeweils in die Auswertung von Reden mit einbezogen.

Als Grundlage für die Auswertung der studentischen Seminarreferate polnischer Sprecher in der hier darzustellenden empirischen Studie (s. Kap. 5) dient der in der rhetorischen Seminarpraxis mittlerweile bewährte Katalog individueller Wirkungskriterien (vgl. Meyer 2013:110-111). Der Katalog umfasst folgende vier Kategorien mit jeweiligen Subkategorien:

- Sprachstil: Wortwahl und Satzbau,

- Denkstil: Redegliederung und -aufbau, Sach- vs. Psychologik (Genauigkeit vs. Einfachheit) sowie Zeitlogik (Angemessenheit bezüglich des Inhalt-Zeit-Verhältnisses),

- Sprechstil: Stimme und Klang, Lautstärke und Akzent, Tempo und Pausen und Lautung,

- Schauform: Mimik und Gestik.

Im Falle der interkulturellen Kommunikation ist mit dem Kriterium des Sprachstils ebenfalls der Aspekt der sprachlichen (lexikalischen und grammatikalischen) Angemessenheit in der Fremdsprache gemeint, mit dem Kriterium des Sprechstils die phonetische Verständlichkeit. Das Kriterium der Schauform wurde zusätzlich um zwei weitere Merkmale ergänzt: Körperhaltung und Raumverhalten. Bei der Auswertung der Referate wurde darüber hinaus ebenfalls die bereits erläuterte Hörerorientierung (Perspektivenübernahme) berücksichtigt.

Im Einzelnen soll ein gutes Seminarreferat u.a. durch folgende Merkmale gekennzeichnet sein: kurze, einfache Sätze, geläufige Wörter bzw. verständlich erklärte Fachbegriffe sowie Fremdwörter (Sprachstil); eine übersichtliche Gruppierung der zusammengehörigen Redeteile (Einleitung, Hauptteil, Schluss), den Überblick über die darzustellenden Inhalte, die Ankündigung der neuen Punkte, Zusammenfassungen in einprägsamen Worten, abschließende Beispiele bzw. Zitate, eine angemessene Länge der einzelnen Referatsteile sowie eine den Hörererwartungen entsprechende Gesamtlänge der Rede (Denkstil); situationsangemessene Betonung, Melodisierung sowie Gliederung durch Pausen, die den freien Vortrag lebendig gestalten lassen und die Behaltensleistung bei den Zuhörern fördern (Sprechstil) sowie eine natürliche, den freien Vortrag unterstützende Gestik, den Kontakt herstellende Mimik (Blickkontakt), eine offene Körperhaltung und nicht zuletzt ein situationsangemessenes Raumverhalten, u.a. einen angemessenen Umgang mit Laptop/Beamer etc. (vgl. auch Allhoff/ Allhoff 2010, Pabst-Weinschenk 2009, Neuber 2013:134-140). 


\section{Zur Untersuchung}

\subsection{Situativer Kontext}

Der situative Kontext der durchgeführten Untersuchung war die institutionelle Lehr-Lern-Kommunikation in ihrer interkulturellen Dimension. Im Fokus stand das studentische Seminarreferat, das zum festen Bestandteil der deutschen akademischen Landschaft gehört und das ebenfalls in den germanistischen Studiengängen in Polen im Rahmen unterschiedlicher Lehrveranstaltungen in der Zielsprache Deutsch eingesetzt wird. So werden die polnischen Studierenden vor die Aufgabe gestellt, insbesondere Inhalte aus ihren Bachelor- bzw. Master-Arbeiten in Form von Seminarreferaten zu präsentieren. Zur Ausführung dieser kommunikativen Aufgabe sollten sie über entsprechende rhetorische Fähigkeiten und Fertigkeiten verfügen, die hier als Redekompetenz aufgefasst wurden (s. Kap. 4).

Unter Berücksichtigung pragmatisch-kommunikativer Faktoren ist das studentische Seminarreferat in Anlehnung an Guckelsberger (2005:27) wie folgt zu charakterisieren: Referate haben die mündliche Wissensvermittlung von Studierenden an Studierende zum Ziel, diese Funktion resultiert aus der traditionellen Rolle der Universität (Kommunikationszweck); der Themenbereich von Referaten wird grundsätzlich durch den inhaltlichen Seminarrahmen vorgegeben (Kommunikationsgegenstand/Inhalt); das Referat wird in der Regel ins Seminar als universitäre Lehrveranstaltung (Kommunikationsort) eingebunden; die Zielgruppe als Publikum sind vor allem Mitstudierende sowie der Dozent (Kommunikationspartner); studentische Referate werden mündlich vorgetragen, wobei in der kommunikativen Wirklichkeit unterschiedliche Präsentationsformen vorliegen, die vom freien Vortragen (ggf. nach einer Textvorlage bzw. Stichpunkten) bis zum wortwörtlichen Ablesen eines ausformulierten Textes reichen; gegenwärtig wird der mündliche Vortrag durch eine Power-Point-Präsentation (als Kommunikationsart) begleitet. Aus sprechwissenschaftlicher Sicht stellt das Referat eine rhetorische Gattung, eine Informationsrede, dar (vgl. z.B. Pabst-Weinschenk 2009:74).

\subsection{Untersuchungsziele}

Die darzustellende Studie ist ein Teil eines größeren Forschungsvorhabens, in dessen Rahmen Wirkungsphänomene, Verständlichkeit sowie die Redekompetenz polnischer Sprecher des Deutschen in der interkulturellen universitären Wissenschaftskommunikation empirisch untersucht wurden. Im Folgenden soll speziell auf zwei Aspekte fokussiert werden: 
- die empirische Erfassung möglicher Verbesserungspotenziale bezüglich der Redekompetenz polnischer Sprecher des Deutschen als Fremdsprache (1),

- die empirische Überprüfung potenzieller funktionaler Abhängigkeiten zwischen der Wirkung der jeweiligen Sprecherperson (deren allgemeiner Eindruck, den sie beim Vortragen auf das Publikum machte) sowie deren Wirkung bezüglich der rhetorischen Kompetenz und bezüglich der Kompetenz im Sprachgebrauchs (2).

Die beiden Aspekte erscheinen vor allem in methodisch-didaktischer Hinsicht durchaus relevant. Das Herausstellen eventueller Verbesserungspotenziale (bzw. Ausbildungslücken) im Bereich einer institutionell bedeutsamen Kompetenz kann nämlich zur Optimierung der Kommunikation in der Fremdsprache Deutsch beitragen. Ebenso kommunikationsfördernd können das Erfassen möglicher Wirkungsvariablen sowie deren funktionale Abhängigkeiten untereinander sein. Sprechwirkungen, die einen festen Bestandteil der rhetorischen Kommunikation ausmachen, sind schließlich für die Erreichung der persuasiven Effekte und somit für die Erreichung der kommunikativen Ziele von Bedeutung.

\subsection{Untersuchungsdesign}

In dem hier dargestellten Versuch wurde eine qualitativ-quantitative Analyse des Testmaterials vorgenommen. Das Testmaterial umfasste im vorliegenden Fall zwei audiovisuelle Aufnahmen studentischer Seminarreferate, die von polnischen Germanistikstudentinnen der Universität Łódź im Rahmen einer Lehrveranstaltung zur Didaktik und Methodik des fremdsprachlichen Unterrichts in der Zielsprache Deutsch gehalten wurden. Die Referate wurden von der Autorin mit einer Kamera und mit einem externen Mikrofon aufgezeichnet und für Untersuchungszwecke entsprechend verarbeitet. Die untersuchten Referate waren jeweils ca. 12 Minuten lang.

Zur Veranschaulichung des breiten Spektrums möglicher Wirkungsvariablen, die in der hier untersuchten Kommunikationssituation (studentisches Seminarreferat) in ihrer interkulturellen Ausprägung jeweils wirksam werden können (s.o.), wurden aus dem gesamten Videokorpus zwei unterschiedliche mündliche Präsentationen wissenschaftlicher Inhalte der Autorin gewählt. Zum einen ging es um ein Referat, das eher schriftsprachlich gestaltet und größtenteils von der Referentin abgelesen wurde, zum anderen handelte es sich um einen weitgehend frei gehaltenen Vortrag. Die beiden Sprecherinnen studierten zur Aufnahmezeit im 6. Semester (B.A.). Das vorausgesetzte Sprachniveau ist C1 nach dem Gemeinsamen Europäischen Referenzrahmen für Sprachen. Die Referentinnen haben jeweils unterschiedlich lang Deutsch als Fremdsprache gelernt und hatten bis zur Aufnahmezeit kein gezieltes Rhetoriktraining (zumindest nicht im Germanistikstudium) absolviert. 
HörerInnen waren in der hier darzustellenden Teiluntersuchung deutsche Studierende am Seminar für Sprechwissenschaft und Phonetik der Martin-Luther-Universität Halle-Wittenberg, zum Teil mit Expertenstatus (d.h. phonetisch und rhetorisch geschult). An der Versuchsrunde nahmen insgesamt bis zu 50 HörerInnen teil. Die Voraussetzung für die Teilnahme am Versuch war Deutsch als Mutter- bzw. Erstsprache. In einem kurzen Fragebogen gaben die meisten ProbandInnen Fremdsprachenkenntnisse an, u.a. des Englischen, Französischen, Italienischen und des Russischen. Die Fragebögen der HörerInnen mit Polnischkenntnissen (als Fremdsprache) wurden bei der Datenauswertung nicht mit berücksichtigt.

Im Hinblick auf die beiden oben genannten Zielsetzungen wurden für die Versuchsrunde zwei verschiedene Typen von Fragebögen konzipiert. Zur Überprüfung der rhetorischen Kompetenz polnischer Sprecher diente als Grundlage der in Kapitel 4 erläuterte Kriterienkatalog des individuellen Wirkungsstils, mit dessen Hilfe einzelne Aspekte von Sprach-, Denk- und Sprechstil sowie Schauform qualitativ analysiert werden konnten. Zur Überprüfung potenzieller funktionaler Abhängigkeiten zwischen der Wirkung polnischer Sprecherinnen (deren allgemeinem Eindruck beim Publikum) sowie deren Wirkung bezüglich der rhetorischen Kompetenz und bezüglich der Kompetenz im Sprachgebrauch wurde hingegen ein Polaritätsprofil in Form einer 6-Stufen-Skala verwendet: ' 1 ' stand entweder für 'positiv' (bei der geschlossenen Frage zur Wirkung der Sprecherperson) oder für 'kompetent' (bei geschlossenen Fragen zur Wirkung der Sprecherperson bezüglich der rhetorischen Gestaltung des Referats und des Sprachgebrauchs), '6' entsprechend für 'negativ' und 'inkompetent'. Die Eintragungen auf der Skala sollten zudem von HörerInnen jeweils begründet bzw. kommentiert werden (eine offene Frage). Diese Kommentare wurden ebenfalls bei der Auswertung berücksichtigt (s.u).

\subsection{Versuchsablauf}

Im Versuchsteil zur rhetorischen Kompetenz der nichtmuttersprachlichen Referentinnen im Deutschen als Fremdsprache arbeiteten zwölf HörerInnen jeweils individuell. Ihre Aufgabe war, die Referate hinsichtlich einzelner Aspekte von Denk-, Sprach- und Sprechstil sowie Schauform qualitativ zu analysieren. Zu diesem Zweck sollten sie sich das Testmaterial beliebig oft anhören/-sehen. Der Versuchsteil zur Wirkung erfolgte dagegen in Gruppen von jeweils 40 Probanden und bestand aus folgenden Schritten: (1) der Vorstellung der allgemeinen Versuchszwecke durch die Autorin, (2) der Verteilung der Fragebögen sowie Fragenklärung, (3) einer standardisierten mündlichen Versuchsanweisung („Gleich wird Ihnen ein 4-Minuten-langer Ausschnitt aus einer Videoaufnahme präsentiert. Sie dürfen sich das Testmaterial nur einmal ansehen/-hören. Markieren Sie bitte 
entsprechende Werte auf der dazugehörigen 6-Stufen-Skala und schreiben Sie jeweils entsprechende Begründungen hinzu. Das dürfen Sie bereits während der Präsentation des Testmaterials sowie in weiteren, dafür vorgesehenen fünf Minuten tun.") und (4) der Versuchsdurchführung. Diese Versuchsrunde nahm insgesamt ca. 12 Minuten in Anspruch.

\subsection{Darstellung und Diskussion der Ergebnisse}

\subsubsection{Zur rhetorischen Kompetenz der polnischen Germanistikstudierenden}

Die qualitative Analyse des Testmaterials wurde von zehn deutschen, phonetisch und rhetorisch geschulten Studierenden durchgeführt. In den dazugehörigen Fragebogen, der in Anlehnung an den Kriterienkatalog des individuellen Wirkungsstils konzipiert wurde, wurden sowohl positive als auch negative Anmerkungen eingetragen, die im Folgenden im Hinblick auf einzelne Subkriterien von Sprach-, Denk-, Sprechstil sowie Schauform (s. Kap. 4) systematisch dargestellt werden. Die negativ formulierten Rückmeldungen werden in der folgenden Übersicht zusätzlich mit einem Minus-Zeichen (-) versehen. Insgesamt handelt es sich dabei um eine Zusammenfassung der in den Fragebögen abgegebenen Kommentare zu den einzelnen Kriterien.

\section{Referentin 1}

Referatstitel: „Lehrperson unter besonderer Berücksichtigung der wichtigsten Berufskompetenzen in Bezug auf den Fremdsprachenunterricht"

\begin{tabular}{|l|l|}
\hline \multicolumn{2}{|c|}{ Sprachstil } \\
\hline Wortwahl & angemessene (geläufige) Wörter, Fachbegriffe erklärt \\
\hline Satzbau & lange Sätze, der schriftsprachliche Stil (-) \\
\hline $\begin{array}{l}\text { Anschaulichkeit } \\
\text { der Darstellung }\end{array}$ & $\begin{array}{l}\text { in dem gesamten Referat eher wenig Beispiele vorhanden, die wenigen } \\
\text { vorhandenen - unzureichend bzw. gar nicht erläutert; es fehlt ein direktes } \\
\text { Ansprechen der Zuhörer (-) }\end{array}$ \\
\hline Art der Darstellung & $\begin{array}{l}\text { eher nüchtern, farblos, unpersönlich, wenig textsortenangemessen; die } \\
\text { Power-Point-Präsentation zu eintönig (die Folien sind zu voll) (-) }\end{array}$ \\
\hline $\begin{array}{l}\text { Gliederung/Ord- } \\
\text { nung }\end{array}$ & $\begin{array}{l}\text { gegliedert, der rote Faden, d.h. die logische Anordnung der Gedanken - } \\
\text { erkennbar }\end{array}$ \\
\hline Kürze/Prägnanz & $\begin{array}{l}\text { aufs Wesentliche beschränkt, aufs Ziel konzentriert, konkret im Thema, } \\
\text { Erläuterungen kurz und prägnant }\end{array}$ \\
\hline Redeaufbau & $\begin{array}{l}\text { die Redeeinleitung vorhanden; kein Überblick am Anfang, jedoch Ziele } \\
\text { und Fragestellungen der Diplomarbeit, zu der gesprochen wird, vorhan- } \\
\text { den; der Redeschluss zwar vorhanden, allerdings eher unbeholfen } \rightarrow \\
\text { auffallende Erleichterung der Sprecherin am Referatsende (-) }\end{array}$ \\
\hline
\end{tabular}




\begin{tabular}{|l|l|}
\hline \multicolumn{2}{|c|}{ Sprechstil } \\
\hline Stimme & $\begin{array}{l}\text { überhöht, angespannt (sehr steifer Oberkörper), brüchig, am Anfang zu } \\
\text { laut } \rightarrow \text { gewisse Aufregung spürbar (-) }\end{array}$ \\
\hline Melodisierung & $\begin{array}{l}\text { monotone Melodieführung } \rightarrow \text { ein Singsang-Effekt, fehlende Lösungs- } \\
\text { tiefe (Stimmhebungen am Ende von Sinnabschnitten) (-); engagiert } \\
\text { (konzentriert), dennoch monoton (-); durch die etwas eintönige Melo- } \\
\text { dieführung und fehlende Lösungstiefe } \rightarrow \text { Eindruck von Ungegliedertheit } \\
\text { (das Zuhören wird erschwert) (-); die an sich langen Sätze wirken auf } \\
\text { Grund fehlender Lösungstiefe noch viel länger (das Zuhören weitgehend } \\
\text { erschwert) (-) }\end{array}$ \\
\hline Betonung & $\begin{array}{l}\text { deutlich zu wenige Satzakzente } \rightarrow \text { fehlende sprecherische Hervorhebung } \\
\text { der Inhalte (-) }\end{array}$ \\
\hline Tempo/Pausen & $\begin{array}{l}\text { zu schnell, zu wenige Pausen, die vorhandenen Pausen durch Zwischenat- } \\
\text { mungen (auch mitten im Satz) produziert, sehr viele störende Füllpausen; } \\
\text { wenig frei gesprochen (-) }\end{array}$ \\
\hline $\begin{array}{l}\text { Mimik/Blickkon- } \\
\text { takt }\end{array}$ & $\begin{array}{l}\text { kaum Blickkontakt mit dem Publikum (Laptop/Notizen) (-); Gesichtsaus- } \\
\text { druck nicht unfreundlich, aber konzentriert }\end{array}$ \\
\hline Gestik & $\begin{array}{l}\text { Gestik nicht untermauernd, verspannt (kaum Gestik), fest am Skript } \\
\text { (Arme zu fest, zu steif) (-) }\end{array}$ \\
\hline Raumverhalten & $\begin{array}{l}\text { Standort zu seitlich, sehr feste Haltung (nicht aufrecht), häufiger Blick auf } \\
\text { Laptop (-) }\end{array}$ \\
\hline
\end{tabular}

\section{Referentin 2}

\section{Referatstitel: „Gruppenarbeit im Fremdsprachenunterricht“}

\begin{tabular}{|c|c|}
\hline \multicolumn{2}{|r|}{ Sprachstil } \\
\hline Wortwahl & $\begin{array}{l}\text { Oberbegriffe erklärt; einige ungewohnte Formulierungen (lexikalische Un- } \\
\text { angemessenheiten) (-) }\end{array}$ \\
\hline Satzbau & $\begin{array}{l}\text { freisprachliches Reden (gesprochene Sprache); Satzstellung oft vertauscht } \\
(-)\end{array}$ \\
\hline $\begin{array}{l}\text { Anschaulichkeit } \\
\text { der Darstellung }\end{array}$ & $\begin{array}{l}\text { lebensnahe Beispiele, direktes Ansprechen der Zuhörer, Fragen beantwor- } \\
\text { ten lassen, Interaktion mit dem Publikum } \rightarrow \text { Reaktionen auf Fragen, Vi- } \\
\text { sualisierungen }\end{array}$ \\
\hline Art der Darstellung & $\begin{array}{l}\text { eine sehr persönliche Darstellung durch den vorhandenen Realitätsbezug, } \\
\text { anregend und interessant; lebensnah und lebendig - aus eigener Erfahrung } \\
\text { vorgetragen; frei gesprochen }\end{array}$ \\
\hline \multicolumn{2}{|r|}{ Denkstil } \\
\hline $\begin{array}{l}\text { Gliederung/Ord- } \\
\text { nung }\end{array}$ & angemessene verständliche Gliederung, einzelne Abschnitte erkennbar \\
\hline Kürze/Prägnanz & einige Abschweifungen (Unwesentliches) (-) \\
\hline Redeaufbau & $\begin{array}{l}\text { ein guter Anfang; ein Überblick am Anfang; kein erkennbarer Schluss (le- } \\
\text { diglich die Klärung von Fragen) (-) }\end{array}$ \\
\hline
\end{tabular}




\begin{tabular}{|l|l|}
\hline \multicolumn{2}{|c|}{ Sprechstil } \\
\hline Stimme & an einigen Stellen zu eng und zu nasal (-); insgesamt angenehm \\
\hline Melodisierung & $\begin{array}{l}\text { eher angemessene Melodisierung (dadurch die sprachliche Unverständlich- } \\
\text { keit gerettet) }\end{array}$ \\
\hline Betonung & $\begin{array}{l}\text { auf Grund des freien Sprechens - eine sehr angemessene Betonung auf } \\
\text { der Satzebene } \rightarrow \text { Sinnkerne angemessen erfasst; die Inhalte entsprechend } \\
\text { hervorgehoben }\end{array}$ \\
\hline Tempo/Pausen & $\begin{array}{l}\text { an einigen Stellen zu schnell,; viele Denkpausen (bei gelesenen Aufzählun- } \\
\text { gen - zu wenig Pausen) (-) }\end{array}$ \\
\hline $\begin{array}{l}\text { Mimik/Blickkon- } \\
\text { takt }\end{array}$ & $\begin{array}{l}\text { natürlicher Blickkontakt, freundliches Gesicht, Lächeln, kontaktfördernder } \\
\text { Gesichtsausdruck }\end{array}$ \\
\hline Gestik & $\begin{array}{l}\text { freie Arme, den Sprech-Denk-Vorgang natürlich unterstützend (auch bei } \\
\text { eigenem Wortfindungsprozess) }\end{array}$ \\
\hline Raumverhalten & lockerer Stand, lockere Körperhaltung (keine Nervosität) \\
\hline
\end{tabular}

Aus den negativen Rückmeldungen, die für die beiden SprecherInnen abgegeben wurden, können folgende Verbesserungspotenziale im Hinblick auf deren rhetorische Kompetenz in der interkulturellen Lehr-Lern-Kommunikation im Bereich der Universität zusammenfassend festgehalten werden:

- die sprachliche Angemessenheit, vor allem auf der grammatikalischen Ebene,

- die Anschaulichkeit und die Art der Darstellung auf der Sprach- und Inhaltsebene,

- die Textsortenangemessenheit bezüglich der sprachlichen Realisierung $\rightarrow$ freies Vortragen erwünscht,

- die rhythmisch-melodische Gestaltung des Vortrags, darunter die melodische Realisierung von Lösungstiefe, sinnvolle Pausensetzung, textsortenangemessenes Sprechtempo, die Angemessenheit bezüglich der Wortbetonung,

- die Reduzierung von Füllwörtern,

- die nonverbale Gestaltung der wissenschaftlichen Präsentation, vor allem in Bezug auf den Einsatz von Gestik, Blickkontakt,

- der weit gefasste Hörerbezug auf der sprachlichen, inhaltlichen, para- und nonverbalen Ebene.

Aus dieser Zusammenfassung wird insgesamt ersichtlich, dass alle Ebenen einer mündlichen Wissenschaftspräsentation ein potenzielles Arbeitsfeld zwecks Verbesserung positiver Kommunikationswirkung auf den Hörer darstellen. Aus dem Vergleich der Rückmeldungen, die jeweils zu den beiden Sprecherinnen abgegeben wurden, ist abzuleiten, dass die zweite Referentin bis auf einige 
wenige Kriterien, wie etwa Kürze/Prägnanz, Tempo/Pausen und vor allem lexikalisch-grammatikalische Angemessenheit im Deutschen als Fremdsprache, im Hinblick auf die rhetorische Kompetenz wesentlich besser ausgefallen ist als die erste Referentin. Zwar hielt diese ihr Referat sprachlich einwandfrei, jedoch entsprach ihr Vortrag lediglich auf der logisch-inhaltlichen Ebene des Denkstils den Erwartungen an ein gutes Seminarreferat (s. Kap. 4). Die negativen Anmerkungen der deutschen HörerInnen betrafen in diesem Fall, wie der Übersicht zu entnehmen ist, vor allem die syntaktischen, para- und nonverbalen Aspekte der mündlichen Präsentation.

\subsubsection{Zur Wirkung der Sprecherpersonen auf die deutschsprachigen HörerInnen}

Auf Grund der quantitativen Datenerhebung mittels eines Polaritätsprofils (einer 6-Stufen-Skala), an der insgesamt 40 deutsche phonetisch und rhetorisch geschulte Studierende (s.o.) teilnahmen, ließen sich folgende Median-Werte ermitteln (in Klammern werden beispielhafte Begründungen der jeweiligen Eintragungen auf der Skala aufgeführt):

\begin{tabular}{|c|c|c|c|}
\hline & $\begin{array}{l}\text { allgemeine Wirkung } \\
\text { der Sprecherperson } \\
\text { '1'-positiv, '6'- } \\
\text { negativ }\end{array}$ & $\begin{array}{l}\text { Wirkung bzgl. } \\
\text { der rhetorischen } \\
\text { Kompetenz } \\
\text { '1'- kompetent, '6'- } \\
\text { inkompetent }\end{array}$ & $\begin{array}{l}\text { Wirkung bzgl. des } \\
\text { Sprachgebrauchs } \\
\text { '1'-kompetent, '6'- } \\
\text { inkompetent }\end{array}$ \\
\hline Referentin 1 & $\begin{array}{l}3 \\
\text { (hat Ahnung vom } \\
\text { Thema, gut vorbereitet, } \\
\text { sympathisch; auch: } \\
\text { Nervosität, schnelles } \\
\text { Sprechtempo, großer } \\
\text { Konzentrationsaufwand } \\
\text { auf der Hörerseite } \\
\text { nötig) }\end{array}$ & $\begin{array}{l}4 \\
\text { (kaum Hörerbezug, } \\
\text { Schriftsprache, } \\
\text { abgelesen bzw. der } \\
\text { Eindruck, als ob sie den } \\
\text { Text auswendig gelernt } \\
\text { hätte) }\end{array}$ & $\begin{array}{l}2 \\
\text { (sichere Wortwahl, keine } \\
\text { Wortfindungsstörungen, } \\
\text { eine sehr gute Grammatik) }\end{array}$ \\
\hline Referentin 2 & $\begin{array}{l}3 \\
\text { (engagiert beim } \\
\text { Vortragen, offene } \\
\text { Ansprechhaltung, nette } \\
\text { Ausstrahlung, sehr } \\
\text { bewegt; dennoch durch } \\
\text { häufige Füllwörter } \\
\text { der Gesamteindruck } \\
\text { verschlechtert) }\end{array}$ & $\begin{array}{l}3 \\
\text { (frei vorgetragen, } \\
\text { authentisch, interessiert, } \\
\text { durch das Fachwissen - } \\
\text { selbstsichere Wirkung; } \\
\text { dennoch auf Grund } \\
\text { des fremden Akzents - } \\
\text { vom Inhalt ablenkend, } \\
\text { irritierend) }\end{array}$ & $\begin{array}{l}3 \\
\text { (sprachliche Unangemes- } \\
\text { senheiten: grammatika- } \\
\text { lische Fehler, untypische } \\
\text { Wörter, insgesamt stok- } \\
\text { kend, Wortfindungs- } \\
\text { schwierigkeite, zahlreiche } \\
\text { Füllwörter) }\end{array}$ \\
\hline
\end{tabular}

Der Übersicht ist Folgendes zu entnehmen: Die beiden Referentinnen wirkten in der rhetorischen Kommunikationssituation studentisches Seminarreferat all- 
gemein eher noch positiv (Median 3), ihre positive Wirkung auf die HörerInnen wurde jedoch auf Grund konkreter Faktoren, wie etwa schnelles Sprechtempo, erkennbare Nervosität bzw. Anhäufung von Füllwörtern, z.T. abgeschwächt. Eher noch positiv wirkte auf die HörerInnen die zweite Referentin auch im Hinblick auf die Kompetenz hinsichtlich der rhetorischen Gestaltung des Referats einerseits und hinsichtlich des Sprachgebrauchs andererseits (Median 3). Bei der ersten Referentin hingegen ist diesbezüglich ein Unterschied festzustellen: Während sie hinsichtlich der rhetorischen Gestaltung des Referats eher inkompetent wirkte (Median 4), wurde ihre sprachliche Kompetenz im Gebrauch lexikalischer und grammatikalischer Mittel wesentlich höher bewertet (Median 2). Dies ist im Allgemeinen auf die Tatsache zurückzuführen, dass sie das Referat wohl nach einem ausformulierten Manuskript gehalten hat. So hatte sie beim Erstellen der Textvorlage zwar mehr Zeit, um über die sprachliche Angemessenheit nachzudenken. Aufgrund der Schriftsprache, die sie in ihrem Vortrag verwendete, kann sogar vermutet werden, dass sie konkrete Textpassagen aus der Fachliteratur wortwörtlich übernahm; dadurch hielt sie jedoch ihr Referat ohne erkennbaren Hörerbezug.

Im Allgemeinen kann am Beispiel der obigen Datenauswertung als Zwischenergebnis festgehalten werden, dass die kommunikative Wirkung polnischer SprecherInnen des Deutschen als Fremdsprache in der universitären Wissenschaftskommunikation durch zahlreiche Variablen geprägt wird, die den positiven Gesamteindruck in der gegebenen Kommunikationssituation entweder abschwächen oder den negativen Gesamteindruck nivellieren. Zu den ersteren Faktoren gehört beispielsweise eine fehlende Hörerorientierung beim Vortragen, zu den letzteren sprachliche Angemessenheit bzw. Sympathie, persönliche Ausstrahlung, Engagement und andere.

\section{Fazit}

Das übergeordnete Ziel der in dem vorliegenden Beitrag dargestellten empirischen Studie war keineswegs festzustellen, ob die Sprecherinnen des Deutschen als Fremdsprache in der universitären Lehr-Lern-Kommunikation rhetorisch kompetent oder inkompetent sind. Eine bloße Feststellung der (In-)Kompetenz der Referentinnen wäre didaktisch-methodisch kaum verwertbar, da sie jeweils zusätzlich durch zahlreiche individuelle, situative, traditionelle und/oder kulturelle Faktoren determiniert ist. Stattdessen wurde darauf abgezielt, die in der Analyse der Seminarreferate aufgedeckten rhetorischen Unzulänglichkeiten der nichtmuttersprachigen Sprecherinnen als mögliche Verbesserungspotenziale zu erfassen. Ihre anschließende Kategorisierung scheint von einem besonderen didaktischen Wert zu sein, da sie einen nachvollziehbaren Ausgangspunkt für eine zielgerichtete Behebung potenzieller Ursachen für negative Kommunikationswirkungen im Rahmen eines interkulturellen Rhetorik-Trainings darstel- 
len kann. Die Ermittlung möglicher Abhängigkeiten zwischen den einzelnen Wirkungsaspekten, die in dem hier behandelten Fall mittels einer quantitativ-qualitativen Datenerhebung erfolgte, erfüllt dabei eine ergänzende Funktion. Die berechneten Mediane wie auch die zu den jeweiligen Eintragungen auf der 6-Stufen-Skala abgegebenen Begründungen veranschaulichen in ihrer Gesamtheit ein breites Spektrum möglicher Wirkungsdeterminanten, die in einem didaktisch gesteuerten Lehr-Lern-Prozess vor dem individuell, situativ und traditionell-kulturell geprägten Hintergrund jeweils mit entsprechender Methodik notwendigerweise zu berücksichtigen sind. Schließlich konnte am Beispiel der empirisch gewonnenen Ergebnisse die wechselseitige Verschränkung von Phonetik, Rhetorik und Persuasion, d.h. der jeweilige Zusammenhang zwischen paraverbaler Gestaltung der Referate, wirksamem Sprechen/Reden und Beeinflussung der Zuhörer in der gegebenen Kommunikationssituation veranschaulicht werden. Dieser Zusammenhang sollte jeweils im Prozess der Vermittlung von Redekompetenz auch in einer Fremdsprache bewusst reflektiert sowie auf der Performanzebene trainiert werden.

\section{Ausblick}

Die aus den empirischen Daten gezogenen Schlussfolgerungen haben zunächst einen weitgehend vorläufigen Charakter und sollen daher an einer anderen Stelle eingehender besprochen werden. Dennoch lässt sich aufgrund der oben angeführten Beispiele aus der Empirie nicht bestreiten, dass die Bewertung der rhetorischen Kompetenz unter Berücksichtigung des Wirkungsfaktors insgesamt einen komplexen Prozess darstellt, in dem jeweils sowohl emotionale als auch kognitive Aspekte ihren Anteil haben. Einerseits handelt es sich um eine unbewusst ablaufende, emotionale Basisbeurteilung im Sinne von gut vs. schlecht hinsichtlich der Zuwendung oder Ablehnung, Sympathie oder Antipathie, ferner auch um eine Kompetenzzuweisung. Andererseits kommt es dabei auf eine durch Normenreflexion entstehende Beurteilung an: Auf Grund konkreter sprachlicher bzw. kultureller Normen werden ebenfalls einzelne Ebenen studentischer Seminarreferate beurteilt. Die als Beurteilungskriterien geltenden Normen sind insbesondere in der interkulturellen Kommunikation ernst zu nehmen, da diesbezügliche Unterschiede zu möglichen Kommunikationsstörungen bzw. -barrieren führen können. Im Hinblick auf mögliche unterschiedliche Bildungstraditionen im Bereich der Vermittlung von Rhetorik wäre u.a. zu überprüfen, welche (und ob überhaupt welche) Aspekte wirksamen Sprechens zum Gegenstand der rhetorischen Sozialisation in der Ausgangssprache werden. Wird beispielsweise dem Schreibprozess mehr Aufmerksamkeit geschenkt, kann es vorkommen, dass der Hörerbezug in der rhetorischen Redepraxis von vorneherein verloren geht. 
Die Bewusstmachung der Komplexität der Vorgänge, die über den Erfolg der jeweiligen Kommunikationssituation entscheiden, ist für den jeweiligen fremdsprachlichen Unterricht von besonderer Relevanz. Diese Erkenntnisse treffen ebenfalls für die Ausbildung der rhetorischen Kompetenz zu, die - wie oben erläutert - über das reine Sprechfertigkeitstraining weit hinausgeht und in der kommunikativen Wirklichkeit als Meilenstein und eine der Schlüsselkompetenzen auf dem beruflichen Werdegang anzusehen ist. Folglich wäre u.a. zu überlegen, auf welche Weise und inwieweit rhetorische Komponenten ebenfalls in die fachbezogenen Seminare sinnvoll integriert werden können, um eine fortlaufende sprachlich-rhetorische Sozialisation der Studierenden sinngemäß zu gewährleisten.

Im Hinblick auf die hier postulierte Einbeziehung der rhetorischen Kompetenz in das Konzept der Schlüsselkompetenzen und somit in die universitären Curricula ist abschließend mit Schwarze (2007:240 - 241) auf einige grundlegende Ansatzpunkte zu verweisen. Bei der Vermittlung der rhetorischen Kompetenz im hochschulischen Rahmen soll zunächst von der empirisch fundierten und nicht der intuitiv-alltagserfahrungsbasierten Kenntnis über die aktuelle Kommunikationsrealität ausgegangen werden. Zu diesem Zweck sind die potenziellen Kommunikationssituationen und Handlungen im Bereich der Universität präzis zu beschreiben. Zugleich soll auch das Wissen über die aktuelle berufliche Realität, dabei speziell die auf dem Arbeitsmarkt gestellten kommunikativen Anforderungen systematisch auf den neuesten Stand gebracht werden. Um dieser Forderung gerecht zu werden, ist insbesondere die gesprächsanalytische Forschung aus dem Bereich der organisationalen Kommunikation, der Kommunikation am Arbeitsplatz bzw. zu Problemen der Wirtschaftskommunikation jeweils zu berücksichtigen. In Anbetracht einer großen Fülle an praktischen Rhetorikratgebern soll nicht zuletzt eine wissenschaftlich untermauerte Positionierung der theoretischen Inhalte aus dem Bereich der rhetorischen Kommunikation angestrebt werden, ohne dass dabei beispielsweise die Hörer-Perspektive bei der Entwicklung der rhetorischen Strategien verloren geht. Somit ließe sich ein gleitender Übergang vom hochschulischen Dasein zur Ausführung von berufsbezogenen Aufgaben sichern.

\section{Literatur}

AlLhOFF Dieter W. / ALLhOFF Waltraud, 2010, Rhetorik \& Kommunikation. Ein Lehr- und Übungsbuch, München.

BARTHEL Henner, 2007, Rhetorische Kompetenz heute? - Zwischenbericht über ein Forschungsprojekt, in: Bose I. (Hrsg.), Sprechwissenschaft: 100 Jahre Fachgeschichte an der Universität Halle, Frankfurt a. M., S. 225-234.

BARTSCH Elmar, 1990, Grundlinien einer „Kooperativen Rhetorik“, in: Geißner Hellmut (Hrsg.), Ermunterung zur Freiheit. Rhetorik und Erwachsenenbildung, Frankfurt a. M., S. 37-49. 
Bose Ines / Schwarze Cordula, 2007, Lernziel Gesprächsfähigkeit im Fremdsprachenunterricht Deutsch, in: Hirschfeld U./Reinke K. (Hrsg.), Phonetik in Deutsch als Fremdsprache: Theorie und Praxis. Zeitschrift für Interkulturellen Fremdsprachenunterricht [Online] 12(2), http:// www.spz.tu-darmstadt.de/projekt_ejournal/jg-12-2/docs/Bose_Schwarze.pdf.

CLYNE Michael, 1993, Pragmatik, Textstruktur und kulturelle Werte. Eine interkulturelle Perspektive, in: Schröder H. (Hrsg.), Fachtextpragmatik, Tübingen, S. $3-18$.

ESSER Ruth, 1997, „Etwas ist mir geheim geblieben am deutschen Referat“. Kulturelle Geprägtheit wissenschaftlicher Textproduktion und ihre Konsequenzen für den universitären Unterricht von Deutsch als Fremdsprache, München.

GEISSNER Hellmut, 1986, Rhetorik und politische Bildung, Frankfurt a. M.

GREINERT Wolf-Dietrich, 2008, Beschäftigungsfähigkeit und Beruflichkeit - zwei konkurrierende Modelle der Erwerbsqualifizierung?, in: Berufsbildung in Wissenschaft und Praxis 37, 4, S. $9-12$.

GUCKELSBERGER Susanne, 2005, Mündliche Referate in universitären Lehrveranstaltungen. Diskursanalytische Untersuchungen im Hinblick auf eine wissenschaftsbezogene Qualifizierung der Studierenden, München.

GUTENBERG Norbert, 2001, Einführung in die Sprechwissenschaft und Sprecherziehung, Frankfurt a. M.

JAKOBS Eva-Maria, 2009, Die Schlüsselqualifikationen Reden und Schreiben in der universitären Ausbildung, in: Fix U./Gardt A./ Knape J. (Hrsg.), Rhetorik und Stilistik. Rhetoric and Stylistics. Ein internationales Handbuch historischer und systematischer Forschung. An International Handbook of Historical and Systematic Research, Berlin/New York, S. 58-68.

KLIEME Eckhard / AVENARIUS Hermann / BLuM Werner et al., 2007, Zur Entwicklung nationaler Bildungsstandards Expertise. Bildungsforschung, Band 1, Berlin (unver. Aufl. 2009), http:// www.bmbf.de/pub/zur_entwicklung_nationaler_bildungsstandards.pdf.

KNAUF Helen, 2001, Schlüsselqualifikationen. Entstehung, Probleme und Relevanz eines Konzeptes, in: Hochschulwesen 2/2001, S. $45-50$.

KRAUS Katrin, 2007, Employability - ein neuer Schlüsselbegriff, in: Panorama. Fachinformationen für Berufsbildung, Berufsberatung und Arbeitsmarkt, S. 4-5 (www.panorama.ch).

MEYer Dirk, 2013, Rede, in: Bose I./Hirschfeld U./Neuber B./Stock E. (Hrsg.), Einführung in die Sprechwissenschaft, Tübingen, S. 121-125.

NeUBER Baldur, 2013, Paraverbale und nonverbale Anteile der rhetorischen Kommunikation, in: Bose I./Hirschfeld U./Neuber B./Stock E. (Hrsg.), Einführung in die Sprechwissenschaft, Tübingen, S. $134-140$.

PABST-WeINSCHENK Marita, 1995, Reden im Studium: Ein Trainingsprogramm, Frankfurt a. M.

PABSt-WeInschenK Marita, 2004, Sprechbildung, in: Pabst-Weinschenk M. (Hrsg.), Grundlagen der Sprechwissenschaft und Sprecherziehung, Basel, S. 14-19.

SCHAPER Niclas, 2012, Fachgutachten zur Kompetenzorientierung in Studium und Lehre. Ausgearbeitet für HRK von Niclas Schaper unter Mitwirkung von Olivier Reis und Johannes Wildt sowie Eva Horvath und Elena Bender, http://www.hrk-nexus.de/fachgutachten_kompetenzorientierung/. 27.02.2016.

SCHWARZE Cordula, 2007, Rhetorik als Schlüsselqualifikation. Überlegungen für die hochschulische Ausbildung, in: Bose I. (Hrsg.), Sprechwissenschaft: 100 Jahre Fachgeschichte an der Universität Halle, Frankfurt a. M., S. 235 - 244.

SCHWARZE Cordula, 2011, Kompetenzorientierung im Bachelor- und Masterstudium Germanistik: Was wollen wir, was können wir?, in: Hackl W./Tanzer U. (Hrsg.), Germanistikstudium in Modulen. Curricula zwischen Berufsorientierung und Fachstudium, Wien, S. 54-77.

SCHWARZE Cordula / BosE Ines, 2013, Mündliche Rhetorik im DaF-Unterricht. Zur Vermittlung von Gesprächs- und Redekompetenz, in: Deutsch als Fremdsprache. Zeitschrift zur Theorie und Praxis des Faches Deutsch als Fremdsprache, Heft 2, S. 74-84. 
Sтоск Eberhard, 1991, Grundfragen der Sprechwirkungsforschung, in: Krech E.-M./Richter G./ Stock E./Suttner J. (Hrsg.), Sprechwirkung: Grundfragen, Methoden und Ergebnisse ihrer Forschung, Berlin, S. 9-58.

WAGNER Roland W., 2004, Grundlagen der mündlichen Kommunikation. Sprechpädagogische Informationsbausteine für alle, die viel und gut reden müssen, Regensburg.

WEINERT Franz E., 2001, Vergleichende Leistungsmessung in Schulen - eine umstrittene Selbstverständlichkeit, in: Weinert F.E. (Hrsg.), Leistungsmessung in Schulen, Weinheim, S. 17-31. 